\title{
TESTES AUTOMATIZADOS PARA CARATERIZAÇÂO DE MATERIAIS MAGNÉTICOS E TRANSFORAMADORES
}

\author{
Angelo M. A. AlmeidA, Benedito A. Luciano, Francisco C. F. Guerra, RAimundo C. S. Freire \\ E-mails: angelo.maxwellegmail.com, beneditoldee.ufcg.edu.br, \\ chagasdee.ufcg.edu.br, freireddee.ufcg.edu.br.
}

\begin{abstract}
Tests on transformers and magnetic materials involve repetitive and time-consuming procedures, when performed manually and on a large scale, can lead to inaccurate results. The task automation instrument control and data display ensures process reliability and agility in the experiments. This paper aims to present an automated test system s used for the characterization of magnetic materials and transformers
\end{abstract}

.Keywords— Magnetic hysteresis, process control, automatized tests, electromagnetic measures.

Resumo- Ensaios em materiais magnéticos e transformadores envolvem procedimentos repetitivos e demorados que, quando realizados manualmente e em larga escala, podem conduzir a resultados imprecisos. A automação de tarefas de controle de instrumentos e exibição de dados garante a confiabilidade do processo e agilidade na realização dos experimentos. Este trabalho tem como objetivo apresentar um sistema de teste s automatizado utilizado para caracterização de materiais magnéticos e transformadores.

Palavras-chave— Histerese magnética, controle de processo, testes automatizados, medidas eletromagnéticas.

\section{Introdução}

Estima-se que nos níveis de transmissão e distribuição as perdas globais (técnicas e comerciais) representem cerca de $14 \%$ de toda a energia gerada no Brasil e que aproximadamente $30 \%$ das perdas técnicas concentrem-se nos núcleos magnéticos dos transformadores de distribuição. Isto representa um enorme prejuízo, que pode ser reduzido com emprego de tecnologias já existentes ou em fase de desenvolvimento.

A partir desta constatação, julgam-se necessárias pesquisas que tratem o problema de modo mais completo e aprofundado, apresentando-se soluções simples e economicamente viáveis. Para alcançar este objetivo, é necessário elaborar um diagnóstico das condições de funcionamento dos componentes do sistema elétrico, segundo os preceitos de eficiência e qualidade de energia.

$\mathrm{Na}$ eletroeletrônica, tem sido observada uma tendência de se substituir os materiais magnéticos convencionais por outros que atendam de modo mais satisfatório não somente os requisitos de eficiência energética, mas também as aplicações em instrumentação. Isto se refere principalmente ao uso de ligas amorfas e nanocristalinas em sensores de alta precisão e em outros dispositivos.

Assim, é indispensável desenvolver instrumentos de elevado grau de exatidão, para uso em atividades de ensino e pesquisa em laboratórios de universidades e institutos, bem como em testes de rotina realizados em instalações elétricas de empresas concessionárias de energia e indústrias. As citadas aplicações se referem tanto a estudos de qualidade de energia quanto a desenvolvimento e aplicação de novos ma- teriais magnéticos. Esta necessidade se justifica pelo fato de que esses equipamentos geralmente apresentam custos elevados, o que suscita a iniciativa de desenvolvê-los através de métodos que os tornem economicamente viáveis para o maior número possível de usuários.

Ensaios em materiais magnéticos e transformadores envolvem procedimentos repetitivos e demorados que, quando realizados manualmente e em larga escala, podem conduzir a resultados imprecisos. A automação de tarefas de controle de instrumentos e exibição de dados garante a confiabilidade do processo e agilidade na realização dos experimentos.

Este trabalho tem como objetivo apresentar um sistema de teste automatizado utilizado para caracterização de materiais magnéticos e transformadores.

\section{Características gerais do sistema}

\subsection{Funções do sistema}

As funções do histeresígrafo são as seguintes:

- traçador de laços $B-H$ (indução versus campo magnético);

- medidor de perdas em materiais magnéticos.

Basicamente, o sistema de medição é composto pelos seguintes elementos:

- microcomputador;

- placa de aquisição de dados;

- gerador de sinais;

- amplificador de potência;

- transformador isolador;

- amplificadores para medição de tensão e de corrente. 
A forma de ligação dos elementos citados no item anterior é mostrada na Figura 1. Dependendo do valor de tensão secundária, o amplificador de tensão pode ser substituído por um divisor resistivo.

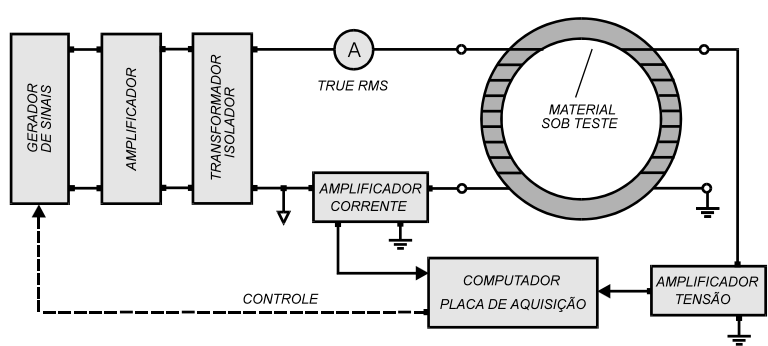

Figura 1. Esquema básico para realização dos ensaios.

\subsection{Descrição dos elementos}

\section{Placa de Aquisição de Dados}

O sistema de aquisição possui uma entrada de tensão e outra de corrente. Os resultados das medições podem ser exibidos na tela do computador. Também podem ser gerados arquivos com a documentação completa das medições e análises realizadas. Neste caso, o sistema de aquisição de dados é capaz de gerar sinais para controlar um gerador de funções que alimenta o sistema sob teste.

\section{Gerador de Sinais}

O gerador de sinais utilizado é do tipo $33210 A$, fabricado pela Agilent Technologies, o qual apresenta os dados fornecidos na Tabela 1.

Tabela 1. Gerador de sinais 33210A, da Agilent Technologies.

\begin{tabular}{|l|c|}
\hline $\begin{array}{c}\text { Grandeza relacionada a formas de } \\
\text { onda senoidais }\end{array}$ & Valores \\
\hline Frequência & $0,1 \mathrm{mHz} \mathrm{a} 10 \mathrm{MHz}$ \\
\hline Resolução de frequência & $1 \mathrm{mHz}$ \\
\hline Distorção harmônica total & $0,04 \%$ \\
\hline Amplitude (50 $\Omega$ ) & $10 \mathrm{mV}$ a $10 \mathrm{~V}$ \\
\hline Amplitude (circuito aberto) & $20 \mathrm{mV}$ a $20 \mathrm{~V}$ \\
\hline
\end{tabular}

\section{Amplificador de Potência}

O amplificador utilizado fornece tensão de saída de 0 a $30 \mathrm{~V} R M S$, e correntes de até $3 \mathrm{~A} R M S$. A faixa de frequências é de zero a $10 \mathrm{kHz}$.

\section{Transformador Isolador}

Esse estágio constitui isolamento elétrico entre o amplificador e o sistema de medição. Também elimina o nível $C C$ presente na tensão de saída do amplificador. $\mathrm{O}$ mesmo possui os seguintes dados nominais de $30 / 30$ V, 3 A RMS.

\section{Amplificador para Medição de Tensão}

Neste amplificador, ilustrado na Figura 2, é utilizado o amplificador de instrumentação INA101, que confere excelente grau de imunidade a ruídos, no caso de medição de sinais de baixa intensidade.

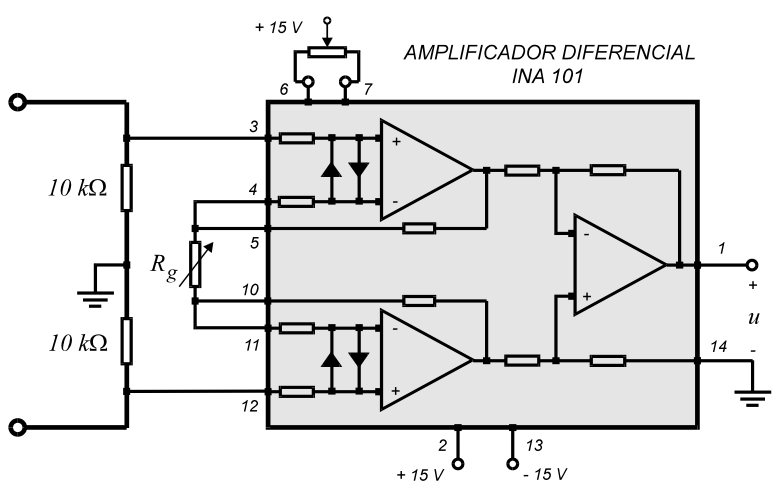

Figura 2. Amplificador para medição de tensão.

O ganho do amplificador é calculado mediante a seguinte expressão:

$$
G=1+\frac{40 k \Omega}{R_{g}},
$$

\section{Amplificador para Medição de Corrente}

O sensor de corrente de alta precisão, semelhante ao apresentado na Figura 2, converte a corrente em tensão proporcional, através do amplificador de instrumentação INA101. É utilizado um resistor shunt entre os pinos 3 e 12, de resistência $60 \mathrm{~m} \Omega$, não indutivo, Esta propriedade é essencial em aplicações de alta frequência, pois as indutâncias parasitas podem se tornar significativas em relação ao baixo valor de resistência. Isto é recomendável quando as correntes são muito pequenas. Neste caso, obtém-se maior grau de exatidão e imunidade a ruídos.

\subsection{Funcionamento do sistema}

O conjunto de hardware e software é utilizado para traçar laços $B-H$ de materiais com amostras em formato de núcleos toroidais, com dois enrolamentos dispostos em torno de si. Como é mostrado na Figura 1 , a intensidade de campo magnético, $H$, é determinada por medição da corrente no enrolamento primário (lado da fonte). A indução magnética, $B$, é determinada mediante integração numérica da tensão no enrolamento secundário, sendo esta operação realizada por função existente no LabVIEW.

Todas as grandezas de entrada e de saída são expressas no Sistema Internacional de Unidades (SI). Esses dados devem ser fornecidos na tela do medidor, mostrada na Figura 3. 


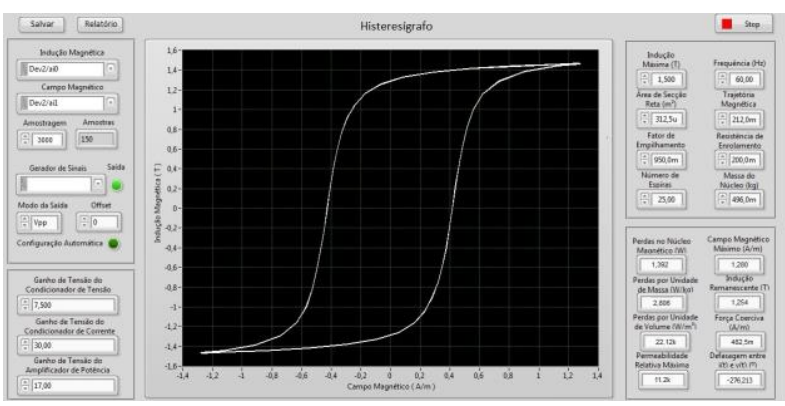

Figura 3. Tela do histeresígrafo com janelas para inserção dos dados de entrada.

O valor de pico da onda de tensão senoidal a ser aplicada no enrolamento primário da amostra de material, $U_{m}$, é previamente calculado pelo software, mediante o emprego da expressão (2), obtido a partir da lei de Faraday:

$$
U_{m}=2 \pi f N F_{e} A B_{m},
$$

Ao calcular esse valor, o computador envia um sinal de controle para o gerador de sinais, fazendo com que este forneça em sua saída uma tensão senoidal com valor de pico igual a esse valor dividido pelo ganho do amplificador de potência, ou seja:

$$
U_{m}^{\prime}=\frac{U_{m}}{G_{P}}
$$

O enrolamento primário passa a ser alimentado por uma tensão senoidal de valor de pico $U_{m}$. Assim, é refletida no enrolamento secundário a tensão a ser amplificada e aplicada num dos canais de entrada da placa de aquisição de dados do medidor, $u_{V}(t)$, o qual calculará os valores da indução magnética mediante a seguinte expressão, na qual $T$ é o período da onda:

$$
B(t)=\frac{1}{N A G_{V}} \int_{t}^{t+T} u_{V}(t) d t
$$

Esse processo de integração numérica é realizado pelo LabVIEW.

O sinal de corrente no enrolamento primário é transformado em uma tensão proporcional $u_{I}(t)$, amplificado e fornecido ao outro canal de entrada analógica da placa de aquisição de dados. Assim, com base na lei circuital de Ampére, a intensidade de campo magnético é dada por:

$$
H(t)=\frac{N u_{I}(t)}{l G_{I}},
$$

Considerando um número de pontos correspondentes ao período da onda de tensão aplicada ao enrolamento primário, as ondas de $B(t)$ e $H(t)$ são compostas de modo a ser exibido o laço $B-H$ na tela do medidor.

A potência média fornecida pela fonte à amostra é dada por:

$$
P=\frac{1}{G_{V} G_{I} T} \int_{t}^{t+T} u_{V}(t) u_{I}(t) d t
$$

O LabVIEW possui funções aritméticas que realizam as operações indicadas na expressão 6. Assim, as perdas no núcleo magnético, $P_{N}$, são dadas por:

$$
P_{N}=P-R I^{2},
$$

A constante $R$ é a resistência do enrolamento primário e $I$ é o valor eficaz da corrente lido pelo amperímetro da Fig. 1.

As grandezas de saída, mostradas na tela do histeresígrafo e em relatório, são indicadas na Tabela 2.

Tabela 2. Grandezas de saída fornecidas pelo histeresígrafo.

\begin{tabular}{|l|c|c|}
\hline \multicolumn{1}{|c|}{ CARACTERÍSTICA } & VALOR & $\mathrm{Hz}$ \\
\hline Perdas no núcleo magnético & 1,385 & $\mathrm{~W}$ \\
\hline Perdas por unidade de volume & $22,001 \times 10^{-3}$ & $\mathrm{~W} / \mathrm{m}^{3}$ \\
\hline Perdas por unidade de massa & 2,792 & $\mathrm{~W} / \mathrm{kg}$ \\
\hline Campo magnético máximo & 1,290 & $\mathrm{~A} / \mathrm{m}$ \\
\hline Indução remanescente & 1,254 & $\mathrm{~T}$ \\
\hline Força coerciva & 0,486 & $\mathrm{~A} / \mathrm{m}$ \\
\hline Permeabilidade relativa máxima & 11037 & - \\
\hline Defasagem entre i 1 (t) e v2 $(\mathrm{t})$ & $-276,200$ & $\circ$ \\
\hline
\end{tabular}

O valor máximo de permeabilidade relativa no núcleo magnético, $\mu_{r m}$, é calculado a partir das permeabilidades diferenciais, determinadas ao longo do ciclo dinâmico $B$ - $H$ registrado, ou seja, calcula-se:

$$
\mu_{k}=\frac{B_{k+1}-B_{k}}{H_{k+1}-H_{k}},
$$

O índice $k$ é a ordem de uma determinada amostra. Efetua-se uma pesquisa para determinação do maior valor de permeabilidade diferencial, $\mu_{k m}$, e calcula-se:

$$
\mu_{r m}=\frac{\mu_{k m}}{\mu_{0}},
$$

A constante $\mu_{0}$ é a permeabilidade magnética no vácuo, igual a $4 \pi \times 10^{-7}$.

\section{Conclusões}

Após a preparação do experimento, com os dados da amostra e condições do ensaio inseridos no software, é possível obter um relatório com as informações apresentadas na Tabela 2. Assim, foi possível caracterizar a amostra de núcleo toroidal em uma única execução do software. 
A utilização de procedimentos semelhantes em larga escala para os ensaios em transformadores pelas concessionárias ou em laboratórios de pesquisa conduz a obtenção de resultados de forma confiável, reduzindo consideravelmente o tempo gasto nos experimentos.

\section{Referências Bibliográficas}

ALMEIDA, A. M. A; GUERRA, F. C. F. FREIRE, R. C. S.; LUCIANO, B. A. (2010). Medidor Virtual para Ensaios em Materiais Magnéticos e Transformadores, XVIII Congresso Brasileiro de Automática, 12 a 16 de setembro de 2010, Bonito - MS.

BANDEIRA, M. I. C. (2004). Automação do Ensaio de Saturação em Transformadores de Corrente Utilizados em Sistemas de Transmissão de Energia Elétrica. Dissertação de mestrado, Programa de Pós-Graduação em Metrologia Científica e Industrial, Universidade Federal de Santa Catarina - UFSC, Florianópolis - SC.

BERTOTTI, G. (1988). General properties of power losses in soft ferromagnetic materials, IEEE Transactions on Magnetics, vol. 24, no. (1), pp. 621-630.

CARMINATI, E.; FERRERO, A. A. (1992). Virtual instrument for the measurement of the characteristics of magnetic materials, IEEE Transactions on Instrumentation and Measurement, vol. 41, no. 6, pp. 1005-1009. DOI: $10.1109 / 19.199382$

GUERRA, F. C. F. (2007). Modelos de Transformador de Corrente para Estudos em Baixas Frequências, Tese de Doutorado, Coordenação de Pós-Graduação em Engenharia Elétrica, Universidade Federal de Campina Grande - UFCG, Campina Grande - PB.

LOPES, V. J. S. (2007). Instrumentação virtual aplicada ao ensino experimental de engenharia elétrica. Dissertação de Mestrado, Escola Politécnica, Universidade de São Paulo.

LUCIANO, B. A.; KIMINAMI, C. S. (2000). Materiais nanocristalinos e suas aplicações na eletroeletrônica, Congresso Brasileiro de Engenharia e Ciência dos Materiais, São Pedro SP.

MOREIRA, A. C. (2006). Uso de Instrumentação Virtual para a Análise da Qualidade da Energia. Dissertação de Mestrado, Faculdade de Engenharia Elétrica e Computação, Universidade Federal de Campinas, Campinas - SP.

NOZAWA, T.; MIISOGAMI, M.; MOGI, H., MATSUO, Y. (1996). Magnetic properties and dynamic domain behavior in grain-oriented $3 \% \mathrm{Si}$ -
Fe, IEEE Transactions on Magnetics, vol. 32, no. 2:, pp. 572-589. DOI: 10.1109/20.486550

SILVA NETO, J. G.; NASCIMENTO, M. M. (2007). Instrumentação Virtual. Curso de Especialização em Instrumentação, Automação, Controle e Otimização de Pro-cessos Contínuos, Escola Politécnica, Universidade Federal da Bahia. Salvador.

PÓLIK, Z.; LUDVIG, T.; KUCSMANN, M. (2007). Measuring of the scalar hysteresis characteristic with a controlled flux density analog an digital integrators. Journal of Electrical Enginnering, vol. 58, no. 4, pp. 236-239.

SHARP, M. R. G.; OVERSHOTT, K. J. (1974). Losses in silicon iron, IEEE Transactions on Magnetics, vol. 10, no. 2), pp. 113-115. DOI: 10.1109/TMAG.1974.1058346

STEGAWSKI, M. A.; SCHAUMANN, R. A. (1998). New virtual-instrumentation-based experimenting environment for undergraduate laboratories with application in research and manufacturing, IEEE Transactions on Instrumentation and Measurement, vol. 47, no. 6, pp.1503-1506.

TRAVIS, J.; KRING, J. (2006). LabVIEW for Everyone: Graphical Programming Made Easy and Fun $-3^{\text {th }}$ Edition, Prentice Hall.

YOSHIZAWA, Y. (1999). Magnetic properties and microstructure of nanocrystalline Fe-based alloys, Materials Science Forum, vol. 307, pp.5162. DOI: 10.4028/www.scientific.net/MSF.307.51 